\title{
Kritik der Verhältnisse und Kritik der Kategorien
}

Zum Tod von Elmar Altvater (1938-2018)

Mit Elmar Altvater, der am 1. Mai im Alter von 79 Jahren seinem Krebsleiden erlag, verliert nicht nur die Linke einen herausragenden Intellektuellen, sondern auch die PROKLA eine Persönlichkeit, die die Zeitschrift Jahrzehnte lang maßgeblich geprägt hat. Elmar hat Probleme des Klassenkampfs, wie die PROKLA bis 1976 hieß, 1971 zusammen mit anderen ehemaligen RedakteurInnen und AutorInnen der Zeitschrift Sozialistische Politik gegründet. Diese war zuvor von einer SEW/DKP-nahen Redaktionsminderheit usurpiert und auf einen politischen Kurs geführt worden, den Elmar und die meisten anderen RedakteurInnen - darunter Christel Neusüß, Wolfgang Schöller, Jürgen Hoffmann und Eckart Hildebrandt - nicht mittragen wollten. Ebenfalls 1971 wurde Elmar, der aus einer Bergarbeiterfamilie in Kamen stammte, in München Soziologie und Ökonomie studiert und an der Universität Erlangen-Nürnberg als wissenschaftlicher Assistent gearbeitet hatte, auf eine Professur für Politische Ökonomie am Otto-Suhr-Institut (OSI) der FU Berlin berufen.

Die frühen 1970er Jahre waren die Zeit, in der eine von Marx inspirierte Analyse der gesellschaftlichen und politischen Verhältnisse an den Universitäten und in den sozialen Bewegungen an Bedeutung gewann. Elmar hatte daran wesentlichen Anteil. In den frühen 1960er Jahren hatte er begonnen, mit Marx - und Marx weiterentwickelnd - die kapitalistischen Gesellschaften ebenso wie jene des „real existierenden Sozialismus“ zu analysieren.
So wurde er zu einer der zentralen Figuren der bundesdeutschen Linken, als die Ende der 1960er aufkommenden sozialen Bewegungen nach Alternativen zu den in den Sozialwissenschaften vorherrschenden Kategorien suchten, um Gesellschaft begreifen und verändern zu können. Die Kritik der Gesellschaft und die Kritik der wissenschaftlichen Kategorien, in denen Gesellschaft gedacht wurde, gingen eine organische Verbindung ein. Wie Elmar in seinem letzten Aufsatz für die PROKLA, der 2007, im Jahr seines Austritts aus der Redaktion, unter dem Titel „Der kurze Sommer des akademischen Marxismus oder: Wie weiter mit der PROKLA?" erschien, resümierend feststellte, war es „die Kritik der realen politischen Verhältnisse“, die die Kritik der Kategorien auslöste, ,in denen die Welt interpretiert wurde. Und erst als man auf Grenzen der Kritikfähigkeit innerhalb des tradierten Kategoriensystems stieß, entstand das Bedürfnis, sich Begriffssysteme, Theorien anzueignen, die an den Universitäten bis dato fast keine Rolle spielten, weil sie ins Abseits gedrängt waren." Die marxsche Theorie wurde so zum zentralen wissenschaftlichen Referenzpunkt. Politisch engagierte sich Elmar im Sozialistischen Büro, später bei den Grünen (die er nach deren Zustimmung zum Kosovokrieg 1999 verließ) und der Linkspartei, vor allem aber für die Gewerkschaften, für die er auch eine Reihe von Studien verfasste.

In der Anfangszeit der PROKLA und seines Wirkens an der FU Berlin leistete Elmar wichtige Beiträge zu der in der 
akademischen Linken intensiv geführten Debatte über den kapitalistischen Staat. Analog zur marxschen Analyse der Wertform ging es ihm und anderen darum, eine Staatsform-Analyse bzw. materialistische Theorie des Staates auszuarbeiten. Was ihn dabei von Beginn an auszeichnete, war das Bemühen um eine zwar theoretisch angeleitete, aber dennoch konkrete Analyse der historischen Erscheinungsformen des Kapitalismus, mit dem Ziel, die Bedingungen für progressive Bewegungen und Politik auszuloten. Dies beinhaltete eine Absage an eine Aneignung der marxschen Theorie als ,ein Dogma, das nur noch 'ex cathedra' auslegebedürftig sei", wie er 1972 in seinem Aufsatz „Zu einigen Problemen des Staatsinterventionismus" in Nr. 3 der PROKLA schrieb. In diesem Sinne entstanden wichtige empirische Analysen zur politischen Ökonomie der Bundesrepublik, so z.B. das zusammen mit Jürgen Hoffmann und Willi Semmler 1979 veröffentlichte Buch Vom Wirtschaftswunder zur Wirtschaftskrise. Darin wird unter anderem am Beispiel der Konzertierten Aktion untersucht, wie staatliche Politik gesellschaftliche Interessen und Konsense mit der Reproduktion des Kapitalverhältnisses kompatibel macht. Wie kaum ein anderer zeigte Elmar früh die Grenzen des Keynesianismus bei dem Versuch auf, dem Kapitalismus einen regulierenden Rahmen zu geben. Seine in diesem Zusammenhang erschienenen Texte, zum Beispiel das gemeinsam mit Kurt Hübner und Michael Stanger 1983 verfasste Buch Alternative Wirtschaftspolitikjenseits des Keynesianismus, zeugen auch von der produktiven Arbeit im Rahmen der PROKLA, denn sie gehen unter anderem auf Arbeiten zurück, die dort zuvor erschienen waren und der Diskussion und Selbstverständigung gedient hatten.

Seit den späten 1960er Jahren trug Elmar entscheidend zu einem kritischen
Verständnis des kapitalistischen Weltmarkts und des Weltwährungssystems bei. Den Grundstein hierfür legte er mit seinem Buch Die Weltwährungskrise von 1969, das methodisch eine zentrale Überlegung enthielt: Der Kapitalismus lässt sich nicht ausgehend vom Nationalstaat und nationalen Markt, sondern nur ausgehend vom Weltmarkt begreifen. In diesem Sinne untersuchte er auch die abhängige Entwicklung des globalen Südens und deren Zuspitzung in der Verschuldungskrise, die vielerorts den Hoffnungen auf eine nachholende, importsubstituierende Industrialisierung ein Ende bereitete. Während in anderen Analysen die beiden Ölpreisschübe der 1970er Jahre als primäre Krisenursache identifiziert wurden, sah Elmar in der Verschuldung des globalen Südens eine Folge der Überakkumulationskrise im globalen Norden. Geldkapital, das in den kapitalistischen Zentren seit Mitte der 1970er Jahre keine hinreichend rentablen produktiven Anlagemöglichkeiten mehr fand, wurde vermittelt über die internationalen Finanzmärkte in den globalen Süden transferiert, von wo aus es in Form von Tilgungen und Zinszahlungen in den Norden zurückfloss. Das Ende des Währungssystems von Bretton Woods und die ihm folgenden Maßnahmen zur Deregulierung und Liberalisierung der Finanzmärkte hatten das institutionelle Umfeld geschaffen, in dem sich die destruktiven Wirkungen einer von der Realökonomie „entkoppelten“ monetären Sphäre entfalten konnten, was sie nach der Wende der US-amerikanischen Notenbank zur Hochzinspolitik, dem sogenannten Volcker-Schock (benannt nach dem damaligen US-Notenbank-Präsidenten), ab 1979 auch taten.

Elmar analysierte diese Zusammenhänge in der PROKLA und am Beispiel Brasiliens in seinem 1987 veröffentlichten 
Buch Sachzwang Weltmarkt, das für die Analyse und Politisierung der Verschuldungskrise im Rahmen der Kampagne gegen das Treffen von Internationalem Währungsfonds (IWF) und Weltbank 1988 in Berlin wichtig war. Mit diesem Buch wandte er sich gleichzeitig intensiver der ökologischen Frage zu, die in der Linken bis dahin ein Schattendasein gefristet hatte, und entwickelte, ausgehend von der marxschen Einsicht in den Doppelcharakter der Ware und des Produktionsprozesses sowie unter Rückgriff auf Nicholas Georgescu-Roegens ökonomische Arbeiten zum Entropiegesetz, eine ökologische Kritik der politischen Ökonomie. Demnach ist kapitalistische Produktion immer zugleich Arbeits- und Verwertungsprozess. Als Verwertungsprozess abstrahiert sie aber von den stofflichen und energetischen Grundlagen, auf die sie als Arbeitsprozess angewiesen ist. Vor allem missachtet sie die Irreversibilität der Transformation von Stoffen und Energie: Die Ressourcen, so heißt es in Sachzwang Weltmarkt, ,sind erschöpflich und knapp und ökonomische Prozesse des Ressourcenabbaus bewirken im System natürlicher Ressourcen irreversible Veränderungen, während im ökonomischen System der Kalkulation von Kapitalwerten und Marktpreisen alle Prozesse [...] zirkulär und reversibel bleiben."

Hierin sah Elmar die ökologische Widersprüchlichkeit des Kapitalismus begründet, die auch in seinen nächsten Büchern (Die Zukunft des Marktes von 1991 und Der Preis des Woblstands von 1993) eine zentrale Rolle spielte. In den verschuldeten Ländern des globalen Südens, die unter dem Diktat des vom globalen Norden dominierten IWF zwecks Devisenerwirtschaftung ihre natürlichen Ressourcen plünderten, manifestierte sich die Widersprüchlichkeit frühzeitig in Gestalt ökologischer Verwüstungen und der damit einhergehenden massiven Menschenrechtsverletzungen. Global, wenn auch immer noch mit höchst unterschiedlichen regionalen Folgen, zeigt sie sich heute an Krisenphänomenen wie dem Klimawandel, die Elmar mit der Kategorie des „Kapitalozän" analysierte - ein Begriff, der die für die sozialökonomische Vermittlung der ökologischen Krise blinde AnthropozänThese vom Kopf auf die Füße stellt. Elmars Einsicht in die destruktiven Kräfte des Kapitalismus begründete eine ähnliche Skepsis hinsichtlich der Möglichkeit, die ökologische Krise unter kapitalistischen Vorzeichen zu überwinden, wie seine weltmarkt- und staatstheoretischen Arbeiten mit Blick auf den Steuerungsoptimismus keynesianischer Wirtschaftstheorie und -politik nahe gelegt hatten. Am Beispiel der 1996 erschienenen, breit rezipierten Studie Zukunftsfähiges Deutschland des Wuppertal Instituts für Klima, Umwelt, Energie deckte Elmar schonungslos die Illusionen auf, denen der Nachhaltigkeitsdiskurs bis heute anhängt. Ist es zulässig, so fragte er in Heft 1/1996 der Blätter für deutsche und internationale Politik, „von ökologischer Nachhaltigkeit zu sprechen und vom Kapitalismus zu schweigen, eine ökologische Revolution [...] einzufordern und politisch, ökonomisch, sozial fast alles beim Alten zu belassen?" Gegenüber dem Zivilgesellschafts-Überschwang der 1990er Jahre, der auch die Studie des Wuppertal Instituts durchzog, plädierte er für "gründliche Erkundungen eines schwierigen Geländes, damit nicht nach dem ersten Schritt die 'ökologiebewußten Menschen', auf die die Studie ihre Hoffnungen setzt, eine frustrierte Kehrtwende machen - zurück in die Sackgasse" - eine Überlegung, die nichts an Aktualität eingebüßt hat. Mit seinen Arbeiten zu Weltmarkt, abhängiger Entwicklung und ökologischer Krise, die auch international breit rezipiert wurden, 
trug Elmar wesentlich dazu bei, den Bewegungen gegen die kapitalistische Globalisierung, wie sie sich in den späten 1990er Jahren herausbildeten, ein begriffliches Instrumentarium zur Verfügung zu stellen. In diesem Zusammenhang sei besonders das Buch Grenzen der Globalisierung hervorgehoben, das er 1996 zusammen mit seiner Lebensgefährtin Birgit Mahnkopf verfasste und das bislang sieben Mal aufgelegt wurde. Elmar und Birgit, die über einen langen Zeitraum hinweg außerordentlich produktiv zusammenarbeiteten, legten darin eine umfassende Analyse vor, die - angeregt sowohl durch Marx als auch durch Polanyi - die globale Entfesselung des kapitalistischen Markts aus allen gesellschaftlichen und ökologischen Bindungen auf den Begriffbrachte. Damals schon identifizierten sie jene Mechanismen, deren Krisenhaftigkeit gut zehn Jahre später im Zusammenbruch der Investmentbank Lehman Brothers kulminierte und die Weltwirtschaft fast in den Abgrund gerissen hätte.

Der produktive organische Zusammenhang zwischen progressiven sozialen Bewegungen und einer einflussreichen akademischen Linken, von dem Elmar geprägt wurde und den er maßgeblich mit prägte, hat sich längst aufgelöst. Der jüngste Bewegungszyklus, jener der globalisierungskritischen Bewegungen der Jahrtausendwende, in denen Elmar als Mitglied im wissenschaftlichen Beirat von Attac selbst mitwirkte, ist abgeebbt, und an den Hochschulen - dies gilt vor allem auch für Elmars Wirkungsstätte, das OSI - ist die Linke marginalisiert. Das hat natürlich auch Konsequenzen für ein
Zeitschriftenprojekt wie die PROKLA, das eben aus diesem organischen Zusammenhang hervorgegangen und in ihm gediehen ist. „Man könnte also zu der Schlussfolgerung gelangen," so schrieb Elmar in dem eingangs bereits zitierten Aufsatz über den kurzen Sommer des akademischen Marxismus, „der Mutterboden einer Zeitschrift wie der PROKLA sei ausgetrocknet und unfruchtbar geworden." Allerdings fügte er gleich hinzu, dass "diese Schlussfolgerung [...] nur teilweise berechtigt" sei, denn nach wie vor existiere eine bunte Vielfalt linker Bewegungen. Die PROKLA müsse sich darin verorten und ,ihre intellektuellen Kapazitäten in ein größeres Projekt wie schon immer mit der gebotenen intellektuellen Distanz und zugleich engagiert einbringen."

Diese Herausforderung nehmen wir als PROKLA-Redaktion an. Und es ist gerade auch Elmars Lebenswerk, das uns helfen wird, sie zu bewältigen und das Projekt einer Kritik der politischen Ökonomie auf der Höhe der Zeit fortzusetzen. Seine hellsichtigen Interventionen, seine intellektuelle Brillanz, sein Humor und seine Menschlichkeit jedoch werden uns dabei fehlen.

\section{Markus Wissen \\ für PROKLA-Redaktion und-Beirat}

In einer der nächsten Ausgaben der PROKLA wird die Redaktion das Werk von Elmar Altvater ausführlicher würdigen, als dies in der kurzen Zeitspanne zwischen seinem Tod und dem Erscheinen dieses Heftes möglich war. 